\title{
A tumour, a cell, a misunderstanding: Trabecular (Merkel cell) carcinoma of the skin
}

\author{
C Urso
}

\begin{abstract}
Trabecular carcinoma has been renamed as Merkel cell carcinoma because, ultrastructurally, tumour cells resemble Merkel cells of the skin. Recently, evidence of exocrine (sweat gland) differentiation in some trabecular carcinomas has been reported. These findings raise two possibilities: (1) trabecular carcinomas are a heterogeneous group of lesions; or (2) that trabecular carcinomas are glandular tumours, in which exocrine features are rarely detected.
\end{abstract}

Trabecular carcinoma, first described by Toker, ${ }^{1}$ has been renamed Merkel cell carcinoma, because, ultrastructurally, tumour cells have neuroendocrine features resembling Merkel cells of the skin.

The Merkel cell is found in the basal layer of the epidermis and in the walls of hair follicles. Trabecular carcinoma arises almost always in the dermis. Therefore, despite some morphological analogies, Merkel cells and trabecular carcinomas are clearly distinguishable by unquestionable differences of site. Moreover, other elements detract from the theory of trabecular carcinomas originating from Merkel cells (Merkel cells contain met-enkephalin, but no neurofilaments; trabecular carcinoma cells contain neurofilaments, but no met-enkephalin). ${ }^{2}$

In 1982 Kroll and Toker found evidence of sweat gland differentiation in two out of 30 cases of trabecular carcinoma. ${ }^{3}$

Of 21 cases of trabecular carcinoma studied

Institute of Anatomic Pathology, University of Florence, Policlinico di Careggi, I-50134 Florence, Italy C Urso

Correspondence to: Dr C Urso

Accepted for publication 20 February 1991 in our Institute in 1985, exocrine differentiation (intercellular and intracytoplasmic glandular lumina and microvilli) was ultrastructurally observed in one case (Abstract; Sixth International Dermatopathology Colloquium, Florence, 1985).

In 1988 Gould et al found two cases showing ductal and tubular structures resembling sweat duct and normal acrosyringium in a series of 42 trabecular carcinomas. Immunocytochemically, ductal cells were positive for CEA and CF-1 (human large molecular weight salivary mucins), antigens specific in the skin for sweat gland structures. In one case squamous differentiation was found. ${ }^{4}$ In a recent study of 18 cases of trabecular carcinoma Heenan et al found one case with intracytoplasmic lumina and microvilli. ${ }^{2}$

These findings suggest two possibilities: (1) trabecular carcinomas are a heterogeneous group of tumours (including a small fraction of lesions with sweat gland differentiation associated with endocrine features); (2) trabecular carcinomas are tumours with both endocrine and exocrine differentiation, in which exocrine features are rarely expressed or scarcely detected. In the latter case trabecular carcinoma should be regarded as a sweat gland neoplasm, as repeatedly proposed by Toker, ${ }^{13}$ or a tumour arising from a stem cell. The presence of squamous differentiation does not conflict with such a view, as squamous features are common in sweat gland carcinoma (malignant acrospiroma, sclerosing sweat duct carcinoma).

The history of interpretation of the histogenesis of trabecular carcinoma shows how a coincidental resemblance associated with a spatial contiguity may lead to misunderstanding.

1 Toker C. Trabecular carcinoma of the skin. Arch Dermatol 1972;105:107-10

2 Heenan PJ, Cole JM, Spagnolo DV. Primary cutaneous neuroendocrine carcinoma (Merkel cell tumor). An adnexal epithelial neoplasm. Am J Dermatopathol 1990;12:7-16.

3 Kroll MH, Toker C. Trabecular carcinoma of the skin. Further clinicopathologic and morphologic study. Arch Pathol Lab Med 1982;106:404-8.

4 Gould E, Albores-Saavedra J, Dubner B, Smith W, Payne CM. Eccrine and squamous differentiation in Merkel cell carcinoma. An immunohistochemical study. Am J Surg Carcinoma. An immun
Pathol 1988;12:768-72. 